\title{
Chaos on compact manifolds: Differentiable synchronizations beyond the Takens theorem
}

\author{
Lyudmila Grigoryeva* \\ Department of Mathematics and Statistics. Universität Konstanz. Box 146. D-78457 Konstanz. Germany. \\ Allen Hart $^{\dagger}$ \\ Department of Mathematical Sciences, University of Bath, Bath BA2 7AY, UK. \\ Juan-Pablo Ortega \\ Division of Mathematical Sciences. Nanyang Technological University. Singapore.
}

\begin{abstract}
This paper shows that a large class of fading memory state-space systems driven by discretetime observations of dynamical systems defined on compact manifolds always yields continuously differentiable synchronizations. This general result provides a powerful tool for the representation, reconstruction, and forecasting of chaotic attractors. It also improves previous statements in the literature for differentiable generalized synchronizations, whose existence was so far guaranteed for a restricted family of systems and was detected using Hölder exponent-based criteria.

Keywords: dynamical systems, synchronization, chaos, attractor, Takens embedding, echo state property, fading memory property, asymptotic stability, echo state network.
\end{abstract}

\section{INTRODUCTION}

Synchronization phenomena between chaotic systems have deserved much attention for decades (see [1-4] for self-contained presentations and many references). This topic has intrinsic theoretical interest and a variety of applications ranging from encryption $[5,6]$ and communication schemes [7] to the analysis of neurological disorders [8, 9]. Generalized synchronizations (GS) were introduced in [10] to describe a system driven by the observations of a chaotic dynamical system that (asymptotically) yields a master-slave configuration. The master is the full dynamical system (not its observations) and the slave is the driven system. Any map that implements this configuration is called a GS.

Given a system driven by the observations of an invertible dynamical system, the main theorem in [11] shows that the asymptotic stability of the system response is a sufficient condition for the existence of a GS. Nevertheless, it was quickly noticed in $[12,13]$ that the GS guaranteed by this theorem may have poor regularity properties, rendering it useless as an attractor representation and reconstruction tool. This fact motivated the distinction in [12] between strong and weak GS depending on whether the synchronization map is differentiable or not. Differentiability was also identified in [13] as a crucial property that determines how useful a GS may be in important tasks such as the estimation of attractor dimensions or Lyapunov exponents. Additionally, a differentiability criterion was introduced in that paper based on the Hölder exponent of the response, mostly

\footnotetext{
* Lyudmila.Grigoryeva@uni-konstanz.de

$\dagger$ A.Hart@bath.ac.uk

‡ Juan-Pablo.Ortega@ntu.edu.sg
}

for invertible driving dynamical systems whose attractors have only one negative Lyapunov exponent.

In this paper we replace the analytical approach in [13] with a geometrical one that, combined with arguments coming from functional analysis, allows us to prove another differentiability criterion. Our main statement (Theorem III.1) shows that if the invertible dynamical system has a compact manifold as phase space and its observations drive a fading memory system with locally state-contracting map, then the generalized synchronizations introduced in [11] always exist and are continuously differentiable. We carefully define all these terms later on in the paper.

Recurrent neural networks and reservoir computing $[14,15]$ have recently enjoyed remarkable success learning [16-19] and classifying [20] chaotic attractors of complex nonlinear infinite dimensional dynamical systems, and detecting GS phenomena [21-23]. This strongly suggested that these machine learning paradigms have Takens embedding-type properties $[24,25]$. This fact has been rigorously established in [26] where the so called Echo State Networks (ESNs) [16, 27-30] driven by onedimensional observations of a given dynamical system on a compact manifold have been shown to produce dynamics that are topologically conjugate to that of the original system. This result is actually proved by, among other things, showing that a natural map that arises in that context (called Echo State Map) is a differentiable generalized synchronization.

Theorem III.1 in this paper is an extensive generalization of that differentiability statement, that is shown to be valid not only for ESNs, but for any fading memory system generated by a locally state-contracting system.

In many of the most recent studies (as in [16-20]), learning and classifying chaotic attractors is tackled by constructing state-space systems to which one naturally associates autonomous systems that serve as proxies for 
the dynamics that needs to be learnt. An important implementation problem that arises using this approach is the so-called curse of dimensionality which, in general, can be elegantly avoided assuming higher order regularity [31]. More specifically, the availability of an injective GS for a dynamical system implies the supervised learnability (explained in the next section) of the dynamics from its observations. The learning is done by approximating a readout function that is defined in a potentially high-dimensional state space. We shall see that the regularity of this readout is determined by the smoothness of the dynamical system in question and of the inverse of the GS (available by the injectivity hypothesis). Hence, if this readout is learnt using standard paradigms like (deep) neural networks or splines, then classical works (see for instance [31, 32]) show that the approximation rate depends on the ratio between the state space dimension (again, potentially very high) and the smoothness of the readout. The differentiability of the GS is therefore of crucial practical importance.

A more recent framework in which GS differentiability arises as a key feature is that of the transfer learning of dynamical systems, as characterized in [33]. This technique translates to the dynamical systems context a concept that has already been successfully used in machine learning [34] and that spells out how to adequately learn the dynamics of a system with training data generated by another one that is close in systems space. When the different systems to be learnt are labeled by a parameters space, a necessary condition for this technique to function is the structural stability of the data generating systems with respect to those parameters, as well as the differentiability of the readouts that are trained to implement the learning. As we explain in the next section, those readouts can be encoded in terms of a GS whose differentiability ensures that of the corresponding readout and hence makes transfer learning possible.

In view of this discussion, this paper identifies a rich class of systems for which continuously differentiable GS are available for invertible dynamical systems on compact manifolds. Additionally, when the GS happens to be injective, the supervised learning of the attractor of a given dynamical system based on finite-length observations is feasible in practice. These results are valuable for the representation, reconstruction, and forecasting of chaotic attractors and explain the excellent performance of reservoir computing and of ESNs in particular evidenced in the above references.

\section{DEFINITIONS AND PRELIMINARY DISCUSSION}

The dynamical system. All along this paper we consider an invertible and discrete-time dynamical system determined by a map $\phi$ that belongs either to the set $\operatorname{Hom}(M)$ of homeomorphisms (continuous invertible maps with continuous inverse) of a compact topologi- cal space $M$ or to the set of diffeomorphisms $\operatorname{Diff}^{1}(M)$ of a finite-dimensional compact manifold $M$. Later on, in the differentiable case, we need to ensure that $M$ can be endowed with a Riemannian metric and that is why we additionally assume that $M$ is connected, Hausdorff, and second-countable (see [35, Proposition $2.10])$. The $d$-dimensional observations of the dynamical system are realized by maps $\omega$ that belong either to $C^{0}\left(M, \mathbb{R}^{d}\right)$ or to $C^{1}\left(M, \mathbb{R}^{d}\right)$ depending on the nature of $\phi\left(\operatorname{Hom}(M)\right.$ or $\operatorname{Diff}^{1}(M)$, respectively). When $M$ is a manifold, the symbol $T M$ denotes the tangent bundle of $M, T \phi: T M \longrightarrow T M$ the tangent map of $\phi$, and $D \omega: T M \longrightarrow \mathbb{R}^{d}$ the differential of the observation map $\omega$.

Sequences. $\mathbb{Z}$ denotes the integers and the symbol $\mathbb{Z}_{-}:=\{\ldots,-2,-1,0\}$ stands for the non-positive integers. Given $D_{d} \subset \mathbb{R}^{d}$, we denote by $D_{d}^{\mathbb{Z}}$ (respectively

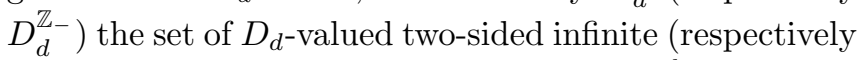
semi-infinite) sequences. The symbol $\left(\ell^{\infty}\left(\mathbb{R}^{d}\right),\|\cdot\|_{\infty}\right)$ (respectively $\left.\left(\ell_{-}^{\infty}\left(\mathbb{R}^{d}\right),\|\cdot\|_{\infty}\right)\right)$ denotes the Banach space of $\mathbb{R}^{d}$-valued two-sided infinite (respectively semi-infinite) sequences that have a finite supremum. For any $t \in \mathbb{Z}$ we define the projection $p_{t}: \ell^{\infty}\left(\mathbb{R}^{d}\right) \longrightarrow \mathbb{R}^{d}$ such that $p_{t}(\mathbf{z}):=\mathbf{z}_{t}$ and the time delay operator $T_{t}: \ell^{\infty}\left(\mathbb{R}^{d}\right) \longrightarrow$ $\ell^{\infty}\left(\mathbb{R}^{d}\right)$ given by $T_{t}(\mathbf{z})_{\tau}:=\mathbf{z}_{\tau-t}, \tau \in \mathbb{Z}$. It is easy to see that both $p_{t}$ and $T_{t}$ are bounded linear operators and that for any $t_{1}, t_{2} \in \mathbb{Z}$ we have

$$
p_{t_{1}+t_{2}}=p_{t_{1}} \circ T_{-t_{2}}=p_{t_{2}} \circ T_{-t_{1}} .
$$

These definitions can be easily adapted to time delays and projections defined in $\ell_{-}^{\infty}\left(\mathbb{R}^{d}\right)$ (see [36, Section 2.3]).

The delay map. Define the $(\phi, \omega)$-delay map $S_{(\phi, \omega)}$ : $M \longrightarrow \ell^{\infty}\left(\mathbb{R}^{d}\right)$ as $S_{(\phi, \omega)}(m):=\left\{\omega\left(\phi^{t}(m)\right)\right\}_{t \in \mathbb{Z}}$ and $S_{(\phi, \omega)}^{-}: M \longrightarrow \ell_{-}^{\infty}\left(\mathbb{R}^{d}\right)$ by $S_{(\phi, \omega)}^{-}(m):=\left\{\omega\left(\phi^{t}(m)\right)\right\}_{t \in \mathbb{Z}_{-}}$. Note that the Abelian group $(\mathbb{Z},+)$ acts both on the phase space $M$ via $\phi$ and on the space $\ell^{\infty}\left(\mathbb{R}^{d}\right)$ via the time delay operators. The map $S_{(\phi, \omega)}$ is equivariant with respect to those two actions, that is,

$$
T_{-t}\left(S_{(\phi, \omega)}(m)\right)=S_{(\phi, \omega)}\left(\phi^{t}(m)\right),
$$

for all $t \in \mathbb{Z}$ and $m \in M$.

State maps, the echo state property (ESP), and the fading memory property (FMP). Let $F: \mathbb{R}^{N} \times$ $\mathbb{R}^{d} \longrightarrow \mathbb{R}^{N}$ be a state map with states in $\mathbb{R}^{N}, N \in$ $\mathbb{N}$. Whenever there exist subsets $D_{N} \subset \mathbb{R}^{N}$ and $D_{d} \subset$ $\mathbb{R}^{d}$ such that $F\left(D_{N} \times D_{d}\right) \subset D_{N}$, we shall denote the restricted state map $F: D_{N} \times D_{d} \longrightarrow D_{N}$ with the same symbol. Given a state map $F: D_{N} \times D_{d} \longrightarrow D_{N}$ and two subsets $V_{d} \subset D_{d}^{\mathbb{Z}}$ and $V_{N} \subset D_{N}^{\mathbb{Z}}$, we say that the state map determined by $F$ has the $\left(V_{d}, V_{N}\right)$-echo state property (ESP) (see [37-39] for in-depth descriptions of this property) when for any $\mathbf{z} \in V_{d} \subset D_{d}^{\mathbb{Z}}$ there exists a unique $\mathbf{x} \in V_{N} \subset D_{N}^{\mathbb{Z}}$ such that

$$
\mathbf{x}_{t}=F\left(\mathbf{x}_{t-1}, \mathbf{z}_{t}\right), \quad \text { for all } t \in \mathbb{Z} .
$$


When the inputs in the system determined by $F$ : $D_{N} \times D_{d} \longrightarrow D_{N}$ are the $\omega$-observations of the dynamical system $\phi$ (we assume that $\omega(\phi(M)) \subset D_{d}$ ) we then talk about the $(\phi, \omega)$-echo state property. This is a particular case of the general definition that we just introduced as the $(\phi, \omega)$-ESP is the same as the $\left(S_{(\phi, \omega)}(M), D_{N}^{\mathbb{Z}}\right)$-ESP in the sense above.

Given a system like (3) that has the $\left(V_{d}, V_{N}\right)$-ESP we can naturally define a causal and time-invariant filter $U^{F}: V_{d} \longrightarrow V_{N}$ by assigning to each $\mathbf{z} \in V_{d}$ the unique $\mathbf{x} \in V_{N}$ that satisfies (3). We recall that the dynamics of causal and time-invariant filters is fully determined by their restriction (in the domain and codomain) to semi-infinite sequences labeled by $\mathbb{Z}_{-}$. We will use exchangeably the filters operating on two-sided infinite and semi-infinite sequences.

Let $U: \ell_{-}^{\infty}\left(\mathbb{R}^{d}\right) \longrightarrow \ell_{-}^{\infty}\left(\mathbb{R}^{N}\right)$ be a causal and timeinvariant filter. Consider a weighting sequence $w$, that is, a strictly decreasing sequence with zero limit $w: \mathbb{N} \longrightarrow$ $(0,1]$ such that $w_{0}=1$, and define $w$-norm by

$$
\|\mathbf{z}\|_{w}:=\sup _{t \in \mathbb{Z}_{-}}\left\{\left\|\mathbf{z}_{t}\right\| w_{-t}\right\}, \quad \text { for any } \quad \mathbf{z} \in\left(\mathbb{R}^{d}\right)^{\mathbb{Z}_{-}} .
$$

We say that $U$ has the fading memory property (FMP) with respect to the sequence $w$ if $U:\left(\ell_{-}^{\infty}\left(\mathbb{R}^{d}\right),\|\cdot\|_{w}\right) \longrightarrow$ $\left(\ell_{-}^{\infty}\left(\mathbb{R}^{N}\right),\|\cdot\|_{w}\right)$ is continuous, that is for any $\mathbf{z} \in \ell_{-}^{\infty}\left(\mathbb{R}^{d}\right)$ and any $\epsilon>0$ there exists a $\delta(\epsilon, \mathbf{z})>0$ such that for any $\overline{\mathbf{z}} \in \ell_{-}^{\infty}\left(\mathbb{R}^{d}\right)$ it holds that

$$
\|\overline{\mathbf{z}}-\mathbf{z}\|_{w}<\delta(\epsilon, \mathbf{z}) \Longrightarrow\|U(\overline{\mathbf{z}})-U(\mathbf{z})\|_{w}<\epsilon .
$$

We recall that for any compact set $D_{d} \subset \mathbb{R}^{d}$ (see [40, Corollary 2.7]) the metric induced by any weighted norm $\|\cdot\|_{w}$ on $D_{d}^{\mathbb{Z}_{-}} \subset \ell_{-}^{\infty}\left(\mathbb{R}^{d}\right)$ generates the product topology on it. The FMP is a dynamic continuity feature that, roughly speaking, makes the influence of the inputs on the outputs of the system determined by $F$ less important as they become more distant in the past.

We shall use the following notation: in Euclidean spaces $\mathbb{R}^{d}$ and for any $L>0$, the symbol $B_{L} \subset \mathbb{R}^{d}$ (respectively $B_{L}(\mathbf{v}) \subset \mathbb{R}^{d}$, with $\mathbf{v} \in \mathbb{R}^{d}$ ) denotes the 2-ball centered at zero (respectively at $\mathbf{v} \in \mathbb{R}^{d}$ ).

The next result shows that locally contracting state maps produce systems that have automatically the ESP and the FMP, provided that the inputs take values in a compact set.

Proposition II.1 Let $F: \mathbb{R}^{N} \times \mathbb{R}^{d} \longrightarrow \mathbb{R}^{N}$ be a continuous state map, let $D_{N} \subset \mathbb{R}^{N}$ be a closed subset, and let $D_{d} \subset \mathbb{R}^{d}$ be a compact subset. Suppose that $F\left(D_{N} \times D_{d}\right) \subset D_{N}$ and that the restriction (denoted with the same symbol) $F: D_{N} \times D_{d} \longrightarrow D_{N}$ is a contraction in the state variable, that is, there exists a constant $0<c<1$ such that for all $\mathbf{x}_{1}, \mathbf{x}_{2} \in D_{N}$ and $\mathbf{z} \in D_{d}$

$$
\left\|F\left(\mathbf{x}_{1}, \mathbf{z}\right)-F\left(\mathbf{x}_{2}, \mathbf{z}\right)\right\| \leq c\left\|\mathbf{x}_{1}-\mathbf{x}_{2}\right\| .
$$

Then: (i) There exists a compact subset $W \subset D_{N}$ such that $F\left(W \times D_{d}\right) \subset W$ and the system determined by $F: W \times D_{d} \longrightarrow W$ with inputs in $D_{d}^{\mathbb{Z}_{-}} \subset \ell_{-}^{\infty}\left(\mathbb{R}^{d}\right)$ has the $\left(D_{d}^{\mathbb{Z}_{-}}, W^{\mathbb{Z}_{-}}\right)$-ESP. This means that for any $\mathbf{z} \in D_{d}^{\mathbb{Z}_{-}}$there exists a unique $\mathbf{x} \in W^{\mathbb{Z}_{-}}$such that

$$
\mathbf{x}_{t}=F\left(\mathbf{x}_{t-1}, \mathbf{z}_{t}\right), \quad \text { for all } t \in \mathbb{Z}_{-} .
$$

If $D_{N}$ is convex then the compact subset $W$ can be chosen to be also convex.

(ii) The recursions (6) determine uniquely a state filter $U^{F}: D_{d}^{\mathbb{Z}_{-}} \longrightarrow W^{\mathbb{Z}_{-}}$that satisfies

$U^{F}(\mathbf{z})_{t}=F\left(U^{F}(\mathbf{z})_{t-1}, \mathbf{z}_{t}\right), \quad$ for all $t \in \mathbb{Z}_{-}$.

The filter $U^{F}$ is continuous when $D_{d}^{\mathbb{Z}_{-}}$and $W^{\mathbb{Z}_{-}}$ are endowed with the product topology. Moreover, $U^{F}$ has the fading memory property with respect to any weighting sequence.

Generalized synchronizations. Consider now a continuously differentiable state map $F: \mathbb{R}^{N} \times \mathbb{R}^{d} \longrightarrow \mathbb{R}^{N}$ with states in $\mathbb{R}^{N}, N \in \mathbb{N}$, as well as the drive-response system associated to the $\omega$-observations of the dynamical system $\phi$ and determined by the recursions:

$$
\mathbf{x}_{t}=F\left(\mathbf{x}_{t-1}, S_{(\phi, \omega)}(m)_{t}\right), \quad t \in \mathbb{Z}, m \in M .
$$

We say that a generalized synchronization (GS) occurs in this configuration when there exists a map $f: M \longrightarrow \mathbb{R}^{N}$ such that for any $\mathbf{x}_{t}, t \in \mathbb{Z}$, as in (8) it holds that

$$
\mathbf{x}_{t}=f\left(\phi^{t}(m)\right)
$$

that is, the time evolution of the dynamical system in phase space (not just its observations) drives the response in (8). All these concepts can be easily extended to the more general situation in which $M$ is just a compact topological space, $\phi \in \operatorname{Hom}(M)$ is a homeomorphism (that is, $\phi$ is continuous, invertible, and the inverse is also continuous), and $\omega$ and $F$ are just continuous.

When a GS is invertible, the readout $h:=\omega \circ \phi \circ f^{-1}$ : $f(M) \subset \mathbb{R}^{N} \longrightarrow \mathbb{R}^{d}$ of the states $\mathbf{x}_{t}$ determined by (9) fully characterize the dynamics of the $\omega$-observations $\left\{\omega\left(\phi^{t}(m)\right)\right\}_{t \in \mathbb{Z}}$ of $\phi$ because $h\left(\mathbf{x}_{t}\right)=\omega \circ \phi\left(f^{-1}\left(\mathbf{x}_{t}\right)\right)=$ $\omega\left(\phi^{t+1}(m)\right)$. This observation implies that this dynamics can be captured via supervised learning if the function $h:=\omega \circ \phi \circ f^{-1}$ is sufficiently regular to be efficiently approximated by a universal family (for instance neural networks or polynomials). This rationale has been profusely exploited in forecasting applications in the context of reservoir computing (see [16-20] as well as [41, 42]). We emphasize that in the reservoir computing context the state map $F$ is quasi-randomly generated and the readout $h$ is constrained to be linear. We are not aware of the existence of a dynamic representation theorem of the type that we just evoked in the presence of invertible 
synchronizations and we conjecture that such a statement can only be formulated up to topological conjugacies, in the spirit of the results in $[26,43]$.

We emphasize that the definition (9) presupposes that $F$ has the $(\phi, \omega)$-echo state property and that $F$ hence determines a unique causal and time-invariant filter $U^{F}: S_{(\phi, \omega)}(M) \longrightarrow\left(\mathbb{R}^{N}\right)^{\mathbb{Z}}$ that associates to each orbit $S_{(\phi, \omega)}(m)$ the unique solution sequence $\mathbf{x} \in\left(\mathbb{R}^{N}\right)^{\mathbb{Z}}$ of (8).

In the following lemma we show that the map $f_{(\phi, \omega, F)}$ : $M \longrightarrow \mathbb{R}^{N}$ defined by

$$
f_{(\phi, \omega, F)}(m):=p_{0}\left(U^{F}\left(S_{(\phi, \omega)}(m)\right)\right),
$$

with $p_{0}:\left(\mathbb{R}^{N}\right)^{\mathbb{Z}} \rightarrow \mathbb{R}^{N}$ the projection onto the zero entry of the sequence, is a GS between the original dynamical system $\phi$ and the response of the system $F$ driven by its $\omega$-observations.

Lemma II.2 Let $\phi \in \operatorname{Hom}(M)$ be an invertible $d y$ namical system on a compact topological space $M, \omega \in$ $C^{0}\left(M, \mathbb{R}^{d}\right)$ an observation map, and $F: D_{N} \times D_{d} \longrightarrow$ $D_{N}$ a continuous state map, with $D_{N} \subset \mathbb{R}^{N}$ and $D_{d} \subset \mathbb{R}^{d}$. If the system determined by $F$ and driven by the $\omega$-observations of $\phi$ has the $(\phi, \omega)-E S P$, then the map $f_{(\phi, \omega, F)}: M \longrightarrow D_{N}$ defined by $f_{(\phi, \omega, F)}(m):=$ $p_{0}\left(U^{F}\left(S_{(\phi, \omega)}(m)\right)\right)$ is a generalized synchronization, that is, it satisfies the defining relation (9) and, more generally,

$$
U^{F}\left(S_{(\phi, \omega)}(m)\right)_{t}=f_{(\phi, \omega, F)}\left(\phi^{t}(m)\right),
$$

for any $t \in \mathbb{Z}, m \in M$.

Lemma II.3 In the same setup as Lemma II.2, the state synchronization map $f_{(\phi, \omega, F)}$ satisfies the identity:

$$
f_{(\phi, \omega, F)}(m)=F\left(f_{(\phi, \omega, F)}\left(\phi^{-1}(m)\right), \omega(m)\right),
$$

for all $m \in M$.

In the next section, the main result of the paper, Theorem III.1, characterizes a large family of state maps $F$ for which the GS $f_{(\phi, \omega, F)}$ is continuously differentiable and, additionally, it is the unique generalized synchronization that satisfies the recursion (12). More specifically, the crucial condition on the state map $F$ that makes GS of the type (10) available is its local contractivity in the state variable. This means that there exists a constant $0<c<1$ and a closed convex set $V \subset \mathbb{R}^{N}$ such that $F(V \times \omega(M)) \subset V$ and, additionally, for all $\mathbf{x}_{1}, \mathbf{x}_{2} \in V$ and $\mathbf{z} \in \omega(M)$,

$$
\left\|F\left(\mathbf{x}_{1}, \mathbf{z}\right)-F\left(\mathbf{x}_{2}, \mathbf{z}\right)\right\| \leq c\left\|\mathbf{x}_{1}-\mathbf{x}_{2}\right\| .
$$

This condition ensures (see Propositions II.1 and D.1) that the ESP always holds. Additionally, we recall that the local contractivity implies the fading memory property (see (4) and part (ii) in Proposition II.1). The FMP implies the so called unique steady-state property, also referred to as input-forgetting property [36, 37]. All these properties are relevant in our context as they all coincide with the asymptotic stability that has been identified in the foundational paper [44] as the characterizing property for the existence of generalized synchronizations.

\section{THE MAIN RESULT}

The next theorem, proved in detail in the appendices (see Proposition D.1, Theorem E.3, and Remark D.2), shows that for invertible dynamical systems $\phi$ defined on compact topological spaces $M$, systems determined by locally contracting state maps $F$ always determine a generalized synchronization that has the same degree of regularity as the dynamical system $\phi$, the system $F$, and the observations $\omega$ that drive it.

Theorem III.1 Let $\phi \in \operatorname{Hom}(M)$ be an invertible discrete-time dynamical system on a compact topological space $M, \omega \in C^{0}\left(M, \mathbb{R}^{d}\right)$ a continuous observation map, and let $F: \mathbb{R}^{N} \times \mathbb{R}^{d} \longrightarrow \mathbb{R}^{N}$ be a continuous state map that is locally contracting in the state variable. Let $V \subset \mathbb{R}^{N}$ be a closed set such that $F(V \times \omega(M)) \subset V$ and where $F$ is state-contracting. Then:

(i) The corresponding restricted system $F: V \times$ $\omega(M) \longrightarrow V$ has the $(\phi, \omega)$-ESP and hence the generalized synchronization $f_{(\phi, \omega, F)}$ is well-defined. In this case, the $G S f_{(\phi, \omega, F)}: M \longrightarrow V$ is continuous and it is the only map with that codomain that satisfies the identity (12).

(ii) The same conclusion holds when $M$ is a compact differentiable manifold, $\phi \in \operatorname{Diff}^{1}(M)$ is an invertible differentiable dynamical system, $\omega$ is of class $C^{1}, F$ is of class $C^{2}$, and $V$ is closed and convex. In this case, if

$$
L_{F_{x}}<\min \left\{1,1 /\left\|T \phi^{-1}\right\|_{\infty}\right\} \text {, }
$$

then the associated GS $f_{(\phi, \omega, F)}$ is continuously differentiable. In (14) $L_{F_{x}}:=\sup _{(\mathbf{x}, \mathbf{z}) \in V \times \omega(M)}\left\{\left\|D_{x} F(\mathbf{x}, \mathbf{z})\right\|\right\}$ and $\|T \phi\|_{\infty}:=\sup _{m \in M}\left\{\left\|T_{m} \phi\right\|\right\}$, with $T_{m} \phi: T_{m} M \longrightarrow$ $T_{\phi(m)} M$ the tangent map of $\phi$ at $m \in M$.

The strategy for obtaining the two parts of this statement consists in using the natural Banach space structures of the spaces $C^{0}\left(M, \mathbb{R}^{N}\right)$ and $C^{1}\left(M, \mathbb{R}^{N}\right)$ of continuous and differentiable functions respectively, to apply the Banach Contraction-Mapping Principle to an automorphism $\Psi: C^{0}\left(M, \mathbb{R}^{N}\right) \longrightarrow C^{0}\left(M, \mathbb{R}^{N}\right)$ of $C^{0}\left(M, \mathbb{R}^{N}\right)$ (respectively of $C^{1}\left(M, \mathbb{R}^{N}\right)$ ) defined using the right hand side of (12), namely,

$$
\Psi(f)(m):=F\left(f\left(\phi^{-1}(m)\right), \omega(m)\right) \text {, for all } m \in M .
$$

The compactness of $M$ is a crucial hypothesis in all these constructions. Using this approach, the local contractivity hypotheses on $F$ (as well as condition (14) in the differentiable case) imply that $\Psi$ is contracting. The generalized synchronization $f_{(\phi, \omega, F)}$ hence arises as its unique fixed point, a condition that amounts to the identity (12). 
It is important to emphasize that for a given state map $F: \mathbb{R}^{N} \times \omega(M) \longrightarrow \mathbb{R}^{N}$ there could exist various disjoint closed sets like $V$ that satisfy the hypotheses in the theorem. The use of the restrictions of $F$ to each of them leads in general to different generalized synchronizations $f_{(\phi, \omega, F)}$ with disjoint codomains. This feature is much related with multistability phenomena and the so-called echo index, as presented in [45]. See also [39, 46] for related discussions.

The strategy followed in the theorem guarantees that $f_{(\phi, \omega, F)}$ can be obtained by iterating the map $\Psi$ using any continuous or differentiable function $f_{0}$ (for instance a constant function) as initial condition. In other words, $f_{(\phi, \omega, F)}$ is the uniform limit of the sequence determined by the iterations:

$$
f_{n+1}=\Psi\left(f_{n}\right), \quad \text { with } \quad f_{0}:=\text { constant } .
$$

There is a fundamental practical difference between the construction of the generalized synchronization using the recursion (15) or via the definition $f_{(\phi, \omega, F)}:=p_{0} \circ U^{F} \circ$ $S_{(\phi, \omega)}$ in (10). The former requires full knowledge about the dynamical system $\phi$ while the latter only uses its $\omega$ observations. This difference is of much relevance when a synchronization has to be constructed or learned using only temporal traces of observations of a given data generating dynamical system.

The existence of differentiable synchronizations for invertible chaotic systems on compact manifolds is not a new fact as it follows from Takens' Theorem [24, 25]. Indeed, this result shows that in the presence of certain non-resonance conditions and for generic scalar observations $\omega \in C^{2}(M, \mathbb{R})$ of a dynamical system $\phi \in$ $\operatorname{Diff}^{1}(M)$, with $M$ compact and $q$-dimensional, a $(2 q+1)$ truncated version $S_{(\phi, \omega)}^{2 q+1}$ of the the $(\phi, \omega)$-delay map given by $S_{(\phi, \omega)}^{2 q+1}(m):=\left(\omega(m), \omega\left(\phi^{-1}(m)\right), \ldots, \omega\left(\phi^{-2 q}(m)\right)\right)$ is a continuously differentiable embedding. This map is in turn the GS corresponding to the linear state map $F(\mathbf{x}, z):=A \mathbf{x}+\mathbf{C} z$, with $A$ the lower shift matrix in dimension $2 q+1$ and $\mathbf{C}=(1,0, \ldots, 0) \in \mathbb{R}^{2 q+1}$ which, by Takens' Theorem, constitutes a differentiable GS for the scalar observations of $\phi$. Theorem III.1 allows us to extend a part of this statement. Indeed, consider an arbitrary linear state map $F(\mathbf{x}, \mathbf{z}):=A \mathbf{x}+\mathbf{C z}, A \in \mathbb{M}_{N, N}$, $\mathbf{C} \in \mathbb{M}_{N, d}$, whose connectivity matrix $A$ has singular values bounded by one, that is $\sigma_{\max }(A)<1$, and that is driven by continuous $d$-dimensional observations $\omega \in C^{0}\left(M, \mathbb{R}^{d}\right)$ of $\phi$. Theorem III.1 guarantees that the system associated to $F$ yields a continuous synchronization $f_{(\phi, \omega, F)}$. Additionally, if $\omega$ is of class $C^{1}$ (not $C^{2}$ as in Takens' Theorem) and $\sigma_{\max }(A)<\min \left\{1,1 /\left\|T \phi^{-1}\right\|_{\infty}\right\}$ then $f_{(\phi, \omega, F)}$ is necessarily differentiable. We note in passing that the encoding of the Takens' delay embedding as a GS shows that the local contractivity invoked in the hypotheses of Theorem III.1 is sufficient but not necessary. Moreover, this result guarantees continuity/differentiability for GSs but does not warrant injectivity or immersivity for which, as we know from the Tak- ens' Theorem case, it is likely to be required additional genericity and/or dimensional conditions.

More recently a similar fact has been proved in [26, Theorem 2.2.2] for systems defined by Echo State Networks, that is, recurrent neural network-like state systems of the form $F(\mathbf{x}, \mathbf{z}):=\boldsymbol{\sigma}(A \mathbf{x}+\mathbf{C z}+\boldsymbol{\zeta})$, where $A \in \mathbb{M}_{N, N}, \mathbf{C} \in \mathbb{M}_{N, d}, \zeta \in \mathbb{R}^{N}$, and the function $\boldsymbol{\sigma}: \mathbb{R}^{N} \longrightarrow \mathbb{R}^{N}$ is constructed by componentwise application of a continuous squashing function $\sigma: \mathbb{R} \longrightarrow \mathbb{R}$. Theorem III.1 guarantees that when $F$ is contracting in the state variable then it yields a continuous synchronization $f_{(\phi, \omega, F)}$. Additionally, if $\omega \in C^{1}\left(M, \mathbb{R}^{d}\right)$, $\sigma \in C^{2}(\mathbb{R})$, and $\sigma_{\max }(A) L_{\sigma}<\min \left\{1,1 /\left\|T \phi^{-1}\right\|_{\infty}\right\}$ with $L_{\sigma}:=\sup _{z \in \mathbb{R}}\left\{\left|\sigma^{\prime}(z)\right|\right\}$, then $f_{(\phi, \omega, F)}$ is necessarily continuously differentiable. This condition coincides with the one in [26, Theorem 2.2.2].

We emphasize that the differentiability results that we have established in this paper obviously do not imply that the generalized synchronizations that we introduced are diffeormorphisms onto their images or even injective. As it was explained in the Introduction and in Section II such feature is very important at the time of using these results to, for instance, learn attractors from time series. It is clear that additional conditions (among others dimensional) need to be required for this to hold. Even though conjectures in this direction have already been formulated (see the last paragraphs in [11]) this question remains open to our knowledge and it will be the subject of a forthcoming publication that is now in preparation [47].

\section{NUMERICAL ILLUSTRATION}

In this Section we illustrate various GSs for the Lorenz system using a state map that satisfies the hypotheses of our main result. We recall that the Lorenz system with the parameter values given in the original paper [48] is determined by the differential equation

$$
\begin{aligned}
\dot{u} & =10(u-v) \\
\dot{v} & =u(28-w)-v \\
\dot{w} & =u v-8 w / 3 .
\end{aligned}
$$

A discrete-time dynamical system $\phi$ can be derived from the Lorenz equation like so:

$$
\phi(u, v, w)=(u, v, w)+\int_{0}^{h}(\dot{u}, \dot{v}, \dot{w}) d t,
$$

with $u(0)=u, v(0)=v, w(0)=w$ and time-step $h$. We simulated a 4000 point (40 time units) trajectory originating from the initial point $m=(0,1,1.05)$ with time-step $h=0.01$. Figure 1 shows this trajectory for times $t \in(20,40)$. If we observe only the $u$ component of this trajectory, then the observation function is $\omega(u, v, w)=u$. The corresponding observed trajectory is illustrated in Figure 2. 
FIG. 1: A trajectory of the Lorenz system.

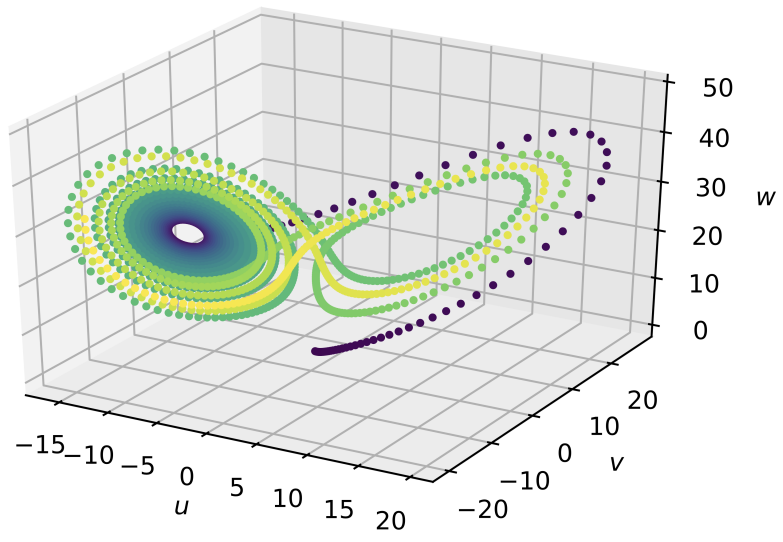

FIG. 2: $u$-component of a trajectory of the Lorenz system.

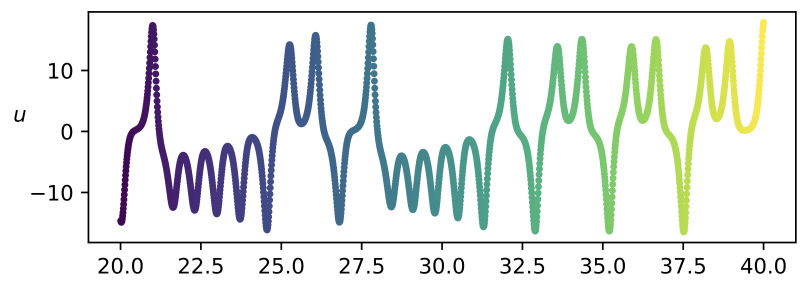
by

We now define the state system $F: \mathbb{R}^{3} \times \mathbb{R} \rightarrow \mathbb{R}^{3}$ given

$$
F(\mathbf{x}, z)=\left(x_{1}^{\alpha}, x_{2}^{\alpha}, x_{3}^{\alpha}\right)+\lambda\left(\sin (k z), \cos (k z), \sin ^{2}(k z)\right)
$$

with $\lambda, k \geq 0$ and $\alpha \in(0,1)$. If we choose $\lambda=0$ then the state system is autonomous and has 8 stable fixed points at $(1,1,1),(-1,1,1),(1,-1,1),(1,1,-1),(-1,-1,1)$, $(-1,1,-1),(1,-1,-1),(-1,-1,-1)$. A cross section of the phase portrait of this autonomous system at the $w=1$ plane is shown in Figure 3 .

If we now choose $\lambda=0.009, k=0.1$ and $\alpha=0.9$, then we can construct a box $V_{1}=[0.9,1.1] \times[0.9,1.1] \times[0.9,1.1]$ containing the fixed point $(1,1,1)$. We can create a similar box about each of the other seven fixed points and denote them $V_{2}, V_{3}, \ldots, V_{8}$. We have by construction for each $i=1, \ldots, 8$ that $F\left(V_{i}, \omega\left(\mathbb{R}^{3}\right)\right) \subset V_{i}$ and that $F$ is state contracting on each box $V_{i}$. Thus, by Theorem III.1 the corresponding restricted state maps $F: V_{i} \times \omega(M) \longrightarrow V_{i}$ have the $(\phi, \omega)$-ESP, so the generalized synchronizations $f_{(\phi, \omega, F)}$ are well-defined and differentiable.

We then computed the states

$$
\mathbf{x}=F\left(\mathbf{x}_{t-1}, \omega\left(\phi^{t}(m)\right)\right)
$$

from two different initial states $\mathbf{x}_{0}=(1,1,1)$ and $\mathbf{x}_{0}=$ $(-1,1,1)$ and plotted the results in Figure 4 . Since $F$ has the fading memory property and hence the inputforgetting property (see Proposition II.1), the values $\mathbf{x}_{t}$ produced by these iterations converge (after a washout
FIG. 3: A phase portrait of the autonomous system $F(\mathbf{x}, z)=\left(x_{1}^{\alpha}, x_{2}^{\alpha}, x_{3}^{\alpha}\right)$ at the cross section $x_{3}=1$ with the 4 stable fixed points.

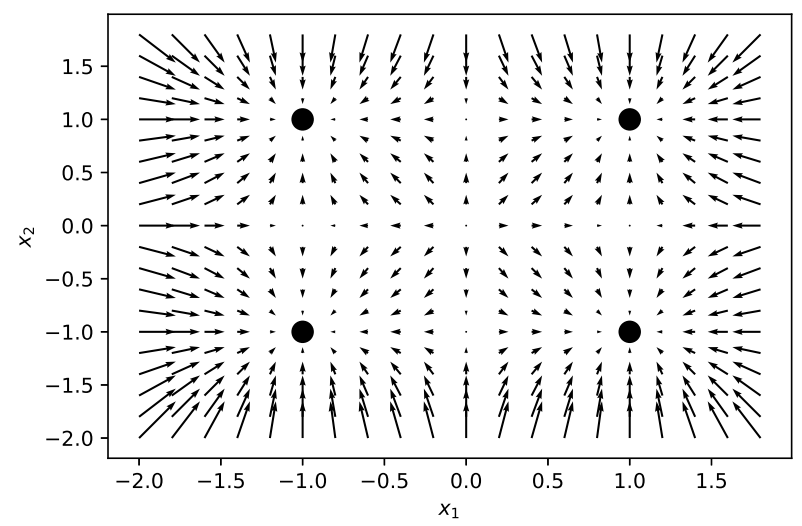

period) to the unique state-space solution $\left\{\mathbf{x}_{t}\right\}_{t \in \mathbb{Z}}$ determined by the infinite input $\left\{\omega\left(\phi^{t}(m)\right)\right\}_{t \in \mathbb{Z}}$. We chose the interval $(0,20)$ as washout period. The defining property (9) of the synchronization maps $f_{(\phi, \omega, F)}$ guarantee that, after the washout period, the values $\mathbf{x}_{t}$ are virtually indistinguishable from the image $f_{(\phi, \omega, F)}\left(\phi^{t}(m)\right)$. Furthermore, for any initial point $x_{0} \in V_{i}$, the states $\mathbf{x}_{t}$ converge to the same image $f_{(\phi, \omega, F)}\left(\phi^{t}(m)\right)$ contained by the box $V_{i}$.

The mapping $f_{(\phi, \omega, F)}$ from the Lorenz attractor to the reservoir space can be numerically computed using two different methods that yield similar results. We have just described the first, and the second uses the iterations (15), taking as an initial conditions the constant map $f_{0}=\mathbf{x}_{0}$, with $\mathbf{x}_{0}=(1,1,1)$ or $\mathbf{x}_{0}=(-1,1,1)$, respectively.

Figure 4 shows that that the GS $f_{(\phi, \omega, F)}$ is visually a differentiable mapping of the Lorenz trajectory into the state space. We stress nevertheless that we have no theoretical guarantees (yet) of the injectivity of $f_{(\phi, \omega, F)}\left(\phi^{t}(m)\right)$ and hence of the supervised learnability of the Lorenz attractor using this particular synchronization.

\section{Appendix A: Proof of Proposition II.1}

The proof of these claims hinges on various results that are already available in the literature.

(i) We start by using an argument similar to the one in [49, Remark 2]. Fix an element $\mathbf{v} \in D_{N}$. Given that $\{\mathbf{v}\} \times D_{d}$ is a compact subset of $\mathbb{R}^{N} \times \mathbb{R}^{d}$ and $F$ is continuous, then (see [50, Theorem 26.5]) $F\left(\{\mathbf{v}\} \times D_{d}\right)$ is a compact subset of $\mathbb{R}^{N}$ and hence bounded (see [50, Theorem 27.3]). This implies the existence of a constant $r>0$ such that $F\left(\{\mathbf{v}\} \times D_{d}\right) \subset \overline{B_{r}(\mathbf{v})} \cap D_{N}$. We now see that for any constant $M>r /(1-c)>0$, the compact 
FIG. 4: Image of the Lorenz solution by two different synchronization maps $f_{(\phi, \omega, F)}$ that contain the points $( \pm 1,1,1)$ in their images.
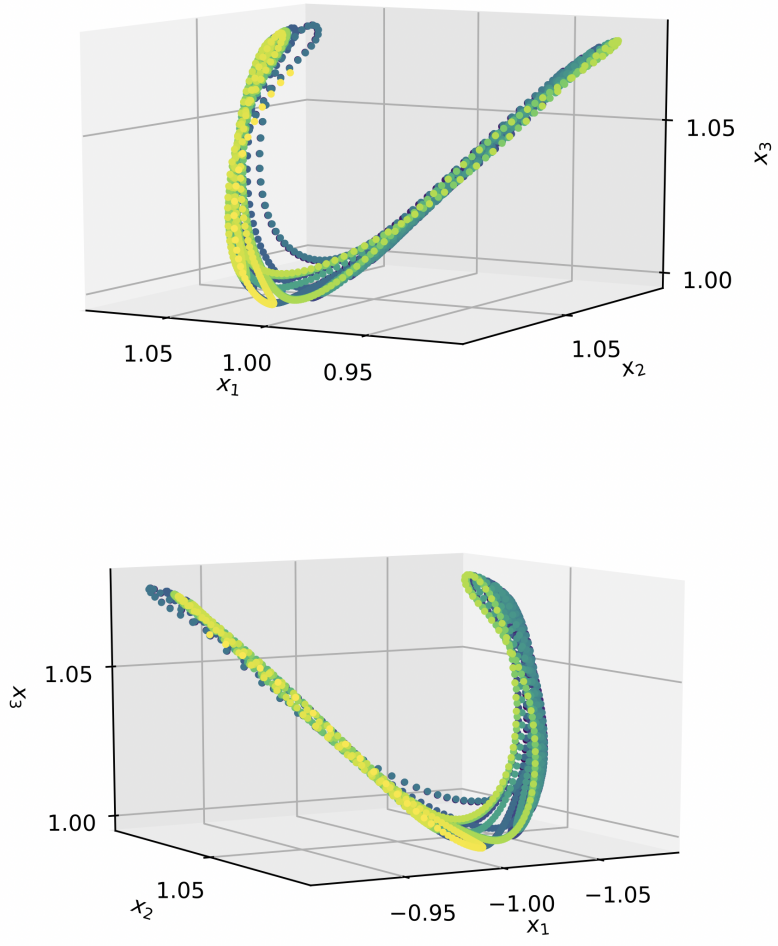

set $W:=D_{N} \cap \overline{B_{M}(\mathbf{v})}$ satisfies that $F\left(W \times D_{d}\right) \subset W$. Indeed, for any $(\mathbf{x}, \mathbf{z}) \in W \times D_{d}$ we can write:

$$
\begin{array}{r}
\|F(\mathbf{x}, \mathbf{z})\| \leq\|F(\mathbf{x}, \mathbf{z})-F(\mathbf{v}, \mathbf{z})\|+\|F(\mathbf{v}, \mathbf{z})\| \\
\leq c\|\mathbf{x}-\mathbf{v}\|+r \leq c M+r<M,
\end{array}
$$

which shows that $F(\mathbf{x}, \mathbf{z}) \in \overline{B_{M}(\mathbf{v})}$. Since by construction $W \subset D_{N}$ and by hypothesis $F\left(D_{N} \times D_{d}\right) \subset D_{N}$ we also have that $F(\mathbf{x}, \mathbf{z}) \in D_{N}$ and hence that $F(\mathbf{x}, \mathbf{z}) \in W$. Notice that if $D_{N}$ is convex then so is $W:=D_{N} \cap \overline{B_{M}(\mathbf{v})}$.

The $\left(D_{d}^{\mathbb{Z}_{-}}, W^{\mathbb{Z}_{-}}\right)$-ESP of $F: W \times D_{d} \longrightarrow W$ and part (ii) can be obtained as in the second part in Theorem 3.1 in [29]. The statement on the continuity with respect to the product topology and the FMP with respect to any weighting sequence is a consequence of Corollary 2.7 in the same reference. Similar results under more or less general hypotheses can also be found in Theorem 7 and Theorem 12 of [36].

\section{Appendix B: Proof of Lemma II.2}

We first recall that, by definition, as $F$ has the $(\phi, \omega)$ ESP, there exists a unique filter $U^{F}: S_{(\phi, \omega)}(M) \longrightarrow$ $\left(D_{N}\right)^{\mathbb{Z}}$ that associates to each orbit $S_{(\phi, \omega)}(m)$ the unique solution sequence $U^{F}\left(S_{(\phi, \omega)}(m)\right) \in\left(D_{N}\right)^{\mathbb{Z}}$ that satisfies

$$
U^{F}\left(S_{(\phi, \omega)}(m)\right)_{t}=F\left(U^{F}\left(S_{(\phi, \omega)}(m)\right)_{t-1}, S_{(\phi, \omega)}(m)_{t}\right),
$$

for all $t \in \mathbb{Z}$. The $(\phi, \omega)$-ESP automatically ensures that $U^{F}$ is causal and time-invariant (see [29, Proposition 2.1] for a detailed proof) and hence

$$
U^{F} \circ T_{t}=T_{t} \circ U^{F}, \quad \text { for all } t \in \mathbb{Z},
$$

with $T_{t}$ the time delay operator introduced above (1). With these elements it is now easy to prove (11). Indeed,

$$
\begin{aligned}
& U^{F}\left(S_{(\phi, \omega)}(m)\right)_{t}=p_{t}\left(U^{F}\left(S_{(\phi, \omega)}(m)\right)\right) \\
& =p_{0} \circ T_{-t}\left(U^{F}\left(S_{(\phi, \omega)}(m)\right)\right)=p_{0}\left(U^{F}\left(T_{-t}\left(S_{(\phi, \omega)}(m)\right)\right)\right) \\
& =p_{0}\left(U^{F}\left(S_{(\phi, \omega)}\left(\phi^{t}(m)\right)\right)\right)=f_{(\phi, \omega, F)}\left(\phi^{t}(m)\right), \quad(\mathrm{B} 3)
\end{aligned}
$$

as required. In this chain of equalities, we have used (1) in the second equality, the time invariance (B2) of $U^{F}$ in the third one, and the time equivariance (2) of $S_{(\phi, \omega)}$ in the fourth one.

\section{Appendix C: Proof of Lemma II.3.}

This is a straightforward corollary of the relation (11) and the recursion (B1) satisfied by $U^{F}$. Indeed, if we set $t=0$ in (11), we have

$$
\begin{aligned}
& f_{(\phi, \omega, F)}(m)=U^{F}\left(S_{(\phi, \omega)}(m)\right)_{0} \\
& =F\left(U^{F}\left(S_{(\phi, \omega)}(m)\right)_{-1}, S_{(\phi, \omega)}(m)_{0}\right) \\
& \quad=F\left(f_{(\phi, \omega, F)}\left(\phi^{-1}(m)\right), \omega(m)\right),
\end{aligned}
$$

where in the second equality we used (B1) and in the third one (11) again with $t=-1$.

\section{Appendix D: Existence and uniqueness of continuous generalized synchronizations}

Proposition D.1 Let $\phi \in \operatorname{Hom}(M)$ be an invertible dynamical system on a compact topological space $M$, $\omega \in C^{0}\left(M, \mathbb{R}^{d}\right)$ an observation map, and let $F: D_{N} \times$ $\omega(M) \longrightarrow D_{N}$ a continuous state map that is a contraction on the state variable, with $D_{N} \subset \mathbb{R}^{N}$ a closed set. Then:

(i) There exists a compact subset $W \subset D_{N}$ such that $F(W \times \omega(M)) \subset W$ and the system determined by $F: W \times \omega(M) \longrightarrow W$ and driven by the $\omega$ observations of $\phi$ has the $(\phi, \omega)$-ESP and hence the generalized synchronization $f_{(\phi, \omega, F)}: M \longrightarrow W$ is well-defined. If $D_{N}$ is convex then $W$ can be chosen to be convex.

(ii) The map $f_{(\phi, \omega, F)}: M \longrightarrow W$ is continuous and it is the only one with that codomain that satisfies the identity (12). 
Proof. (i) This claim is a consequence of Proposition II.1. Indeed, since $\omega$ is continuous and $M$ is compact then so is $\omega(M)$. The contractivity hypothesis and the closeness of $D_{N}$ imply by Proposition II.1 the existence of the compact set $W$ in the statement and that the corresponding system $F: W \times \omega(M) \longrightarrow W$ has the $\left(\omega(M)^{\mathbb{Z}_{-}}, W^{\mathbb{Z}_{-}}\right)$-ESP. Given that $S_{(\phi, \omega)}^{-}(M) \subset \omega(M)^{\mathbb{Z}_{-}}$ this implies that the $\left(S_{(\phi, \omega)}^{-}(M), W^{\mathbb{Z}_{-}}\right)$-ESP also holds. This condition amounts to the $(\phi, \omega)$-ESP and to the existence of a FMP filter $U^{F}: S_{(\phi, \omega)}^{-}(M) \longrightarrow W^{\mathbb{Z}_{-}}$which, in passing, shows that the generalized synchronization $f_{(\phi, \omega, F)}:=p_{0} \circ U^{F} \circ S_{(\phi, \omega)}: M \longrightarrow W$ is well-defined.

(ii) We first show the continuity of $f_{(\phi, \omega, F)}:=p_{0} \circ$ $U^{F} \circ S_{(\phi, \omega)}: M \longrightarrow W$ by noticing that it is a composition of continuous functions. Indeed, using the notation introduced in the previous point, if we endow the sets $\omega(M)^{\mathbb{Z}_{-}}$and $W^{\mathbb{Z}_{-}}$with the product topology, the map $p_{0}: W^{\mathbb{Z}_{-}} \longrightarrow W$ is clearly continuous, $U^{F}: \omega(M)^{\mathbb{Z}_{-}} \longrightarrow W^{\mathbb{Z}_{-}}$is continuous by Proposition II.1, and $S_{(\phi, \omega)}: M \longrightarrow \omega(M)^{\mathbb{Z}_{-}}$is also continuous because it is a Cartesian product of continuous maps (see [50, Theorem 19.6]).

In order to prove the uniqueness statement, we first endow the set of continuous functions $C^{0}\left(M, \mathbb{R}^{N}\right)$ with the Banach space structure induced by the norm

$$
\|f\|_{\infty}:=\sup _{m \in M}\{\|f(m)\|\}, \quad f \in C^{0}\left(M, \mathbb{R}^{N}\right) .
$$

We emphasize that this norm is always finite due to the compactness of $M$. Moreover, it is easy to show that the subset $C^{0}(M, W)$ of $C^{0}\left(M, \mathbb{R}^{N}\right)$ made of functions that have the compact subset $W \subset \mathbb{R}^{N}$ as codomain is closed with respect to the topology generated by the norm $\|\cdot\|_{\infty}$. Indeed, let $f \in C^{0}\left(M, \mathbb{R}^{N}\right)$ be an element in the closure of $C^{0}(M, W)$ and let $\left\{f_{n}\right\}_{n \in \mathbb{N}}$ be a sequence of elements in $C^{0}(M, W)$ that have $f$ as limit. This implies that for any $\epsilon>0$ there exists $N(\epsilon) \in \mathbb{N}$ such that for any $n>N(\epsilon)$ we have that $\left\|f_{n}-f\right\|_{\infty}<\epsilon$. This implies, in particular, that for any point $m \in M$ :

$$
\left\|f_{n}(m)-f(m)\right\| \leq\left\|f_{n}-f\right\|_{\infty}<\epsilon
$$

and hence $\lim _{n \rightarrow \infty} f_{n}(m)=f(m)$, which shows that $f(m)$ is a limit point of $W$ and hence belongs to $W$ as it is a closed set. Since $m \in M$ is arbitrary this shows that $f \in$ $C^{0}(M, W)$ and hence $C^{0}(M, W)$ is closed in $C^{0}\left(M, \mathbb{R}^{N}\right)$.

We now shall prove the uniqueness statement by showing that the map $\Psi: C^{0}(M, W) \longrightarrow C^{0}(M, W)$ defined by

$$
\Psi(f)(m):=F\left(f\left(\phi^{-1}(m)\right), \omega(m)\right),
$$

for all $m \in M, f \in C^{0}\left(M, \mathbb{R}^{N}\right)$, is well-defined and a contraction with respect to the norm introduced in (D1). The result follows then from the Banach ContractionMapping Principle (see [51, Theorem 3.2]).

First, the continuity hypotheses on $\phi, \omega$, and $F: W \times$ $\omega(M) \longrightarrow W$ imply that $\Psi(f) \in C^{0}(M, W)$ whenever $f \in C^{0}(M, W)$. Now, let $f, g \in C^{0}(M, W)$. Then, since we assume that $F$ is a contraction in the state variable with constant $0<c<1$, we have that,

$$
\begin{gathered}
\|\Psi(f)-\Psi(g)\|_{\infty} \\
=\sup _{m \in M}\left\{\| F\left(f\left(\phi^{-1}(m)\right), \omega(m)\right)\right. \\
\left.-F\left(g\left(\phi^{-1}(m)\right), \omega(m)\right) \|\right\} \\
\leq c \sup _{m \in M}\left\{\left\|f\left(\phi^{-1}(m)\right)-g\left(\phi^{-1}(m)\right)\right\|\right\}=c\|f-g\|_{\infty},
\end{gathered}
$$

which shows that $\Psi$ is a contraction with respect to the norm in (D1) and hence there exists a unique element in $C^{0}(M, W)$ that satisfies the identity (12). As we just proved that $f_{(\phi, \omega, F)} \in C^{0}(M, W)$ and by Lemma II.3 the state synchronization map $f_{(\phi, \omega, F)}$ satisfies (12), that unique element is $f_{(\phi, \omega, F)}$ necessarily.

Remark D.2 It is worth emphasizing that, for a given state map $F: \mathbb{R}^{N} \times \omega(M) \longrightarrow \mathbb{R}^{N}$, there could exist various disjoint closed sets like $D_{N}$ that satisfy the hypotheses of the proposition. The use of the restrictions $F: D_{N} \times \omega(M) \longrightarrow D_{N}$ to each of them leads in general to different generalized synchronizations $f_{(\phi, \omega, F)}$ whose codomains are compact subsets of each of the closed set $D_{N}$. This feature is much related with multistability phenomena and the so-called echo index, as presented in [45]. See also $[39,46]$ for related discussions.

Remark D.3 Having obtained in the proof the state synchronization map $f_{(\phi, \omega, F)}: M \longrightarrow W$ as the unique fixed point of a contracting map, a standard result about maps of this type on metric spaces (see [51, Proposition 3.4]), guarantees that $f_{(\phi, \omega, F)}$ can be obtained by iterating the map $\Psi$ defined in (D2) using any function $f_{0} \in C^{0}(M, W)$ (for instance a constant function) as initial condition. In other words, $f_{(\phi, \omega, F)}$ is the uniform limit of the sequence of functions determined by the iterations:

$$
f_{n+1}=\Psi\left(f_{n}\right), \quad \text { with } \quad f_{0}:=\mathbf{w} \in W,
$$

where the constant element $\mathbf{w} \in W$ is arbitrary.

Remark D.4 There is a fundamental difference of much practical importance between the construction of the generalized synchronization $f_{(\phi, \omega, F)}$ via the iteration of the map $\Psi$ as in (D4) and using the definition $f_{(\phi, \omega, F)}:=$ $p_{0} \circ U^{F} \circ S_{(\phi, \omega)}$. The former requires full knowledge about the dynamical system $\phi$ while the latter only uses its $\omega$ observations. This difference is of much relevance when a synchronization has to be constructed or learned using only observations of a given data generating dynamical system. 


\section{Appendix E: Existence and uniqueness of differentiable synchronizations}

In this section we extend the result in Proposition D.1 and we show that when the dynamical system $\phi \in$ $\operatorname{Diff}^{1}(M)$ and the maps $\omega$ and $F$ are differentiable then the state synchronization map $f_{(\phi, \omega, F)}$ is necessarily differentiable. The strategy that we follow also consists in proving that the map $\Psi$ introduced in (D2) is a contraction but, this time around, on a well-chosen closed subset of $C^{1}\left(M, \mathbb{R}^{N}\right)$. We start with some preliminaries.

a. Banach space structures in $C^{1}\left(M, \mathbb{R}^{N}\right)$. All along this section we assume that $M$ is a compact, connected, Hausdorff, and second-countable manifold and hence it can be endowed with a Riemannian metric $g$ (see [35, Proposition 2.10]). Now, for any $f \in C^{1}\left(M, \mathbb{R}^{N}\right)$, define

$$
\begin{gathered}
\|D f\|_{\infty}=\sup _{m \in M}\{\|D f(m)\|\} \quad \text { with } \\
\|D f(m)\|=\sup _{\substack{\mathbf{v} \in T_{m} M \\
\mathbf{v} \neq \mathbf{0}}}\left\{\frac{\|D f(m) \cdot \mathbf{v}\|}{(g(m)(\mathbf{v}, \mathbf{v}))^{1 / 2}}\right\} .
\end{gathered}
$$

Analogously, if $\phi: M \rightarrow M$ is a $C^{1}$ map, we can define:

$$
\begin{gathered}
\|T \phi\|_{\infty}=\sup _{m \in M}\left\{\left\|T_{m} \phi\right\|\right\} \quad \text { with } \\
\left\|T_{m} \phi\right\|=\sup _{\substack{\mathbf{v} \in T_{m} M \\
\mathbf{v} \neq \mathbf{0}}}\left\{\frac{\left(g(\phi(m))\left(T_{m} \phi \cdot \mathbf{v}, T_{m} \phi \cdot \mathbf{v}\right)\right)^{1 / 2}}{(g(m)(\mathbf{v}, \mathbf{v}))^{1 / 2}}\right\} .
\end{gathered}
$$

It can be proved by using the results in Chapter 2 of [52] that, for any $\delta>0$, the norms $\|\cdot\|_{C^{1}(\delta)}$ defined by

$$
\|f\|_{C^{1}(\delta)}:=\|f\|_{\infty}+\delta\|D f\|_{\infty}
$$

endow $C^{1}\left(M, \mathbb{R}^{N}\right)$ with a Banach space structure. It can also be shown (see [52, Theorem 11.2 (ii)]) that all these norms generate the same topology in $C^{1}\left(M, \mathbb{R}^{N}\right)$ that is also independent of the choice of Riemannian metric $g$ and coincides with the weak and strong topologies introduced in Chapter 2 of [53].

We introduce two technical lemmas that are needed later on.

Lemma E.1 In the setup that we just described, let $R>$ $0, W \subset \mathbb{R}^{N}$ a closed set, and define:

$$
\Omega(R, W):=\left\{f \in C^{1}(M, W) \mid\|D f\|_{\infty} \leq R\right\},
$$

where $f \in C^{1}(M, W)$ is by definition the subset of $f \in C^{1}\left(M, \mathbb{R}^{N}\right)$ made by the functions which take values in $W \subset \mathbb{R}^{N}$. The set $\Omega(R, W)$ is closed in $\left(C^{1}\left(M, \mathbb{R}^{N}\right),\|\cdot\|_{C^{1}(\delta)}\right)$ for any $\delta>0$.
Proof. We proceed by showing that $\Omega(R, W)$ is the intersection of two closed sets, that is, $\Omega(R, W)=\Omega(R) \cap C^{1}(M, W)$, where $\Omega(R):=$ $\left\{f \in C^{1}\left(M, \mathbb{R}^{N}\right) \mid\|D f\|_{\infty} \leq R\right\}$.

We first prove that $\Omega(R)$ is closed by showing that the complementary set $\Omega(R)^{c}$ is open in $\left(C^{1}\left(M, \mathbb{R}^{N}\right),\|\cdot\|_{C^{1}(\delta)}\right)$. Let $f \in \Omega(R)^{c}$ such that $\|D f\|_{\infty}=K>R$. Given $\epsilon:=\delta(K-R)$ we will show that the ball $B_{C^{1}(\delta)}(f, \epsilon)$ is a subset of $\Omega(R)^{c}$. Indeed, for any $g \in B_{C^{1}(\delta)}(f, \epsilon)$ :

$$
\begin{gathered}
K=\|D f\|_{\infty}=\|D f+D g-D g\|_{\infty} \\
\leq\|D f-D g\|_{\infty}+\|D g\|_{\infty} \\
\leq \frac{1}{\delta}\|f-g\|_{\infty}+\|D f-D g\|_{\infty}+\|D g\|_{\infty} \\
=\frac{1}{\delta}\|f-g\|_{C^{1}(\delta)}+\|D g\|_{\infty}<\frac{\epsilon}{\delta}+\|D g\|_{\infty} \\
=K-R+\|D g\|_{\infty},
\end{gathered}
$$

which implies that $\|D g\|_{\infty}>R$ and hence that $B_{C^{1}(\delta)}(f, \epsilon) \subset \Omega(R)^{c}$.

We now show that $C^{1}(M, W)$ is closed in $C^{1}\left(M, \mathbb{R}^{N}\right)$. Let $f \in C^{1}\left(M, \mathbb{R}^{N}\right)$ be an element in the closure of $C^{1}(M, W)$ and let $\left\{f_{n}\right\}_{n \in \mathbb{N}}$ be a sequence of elements in $C^{1}(M, W)$ that have $f$ as limit. This implies that for any $\epsilon>0$ there exists $N(\epsilon) \in \mathbb{N}$ such that for any $n>N(\epsilon)$ we have that $\left\|f_{n}-f\right\|_{C^{1}(\delta)}<\epsilon$. This implies, in particular, that for any point $m \in M$ :

$$
\begin{aligned}
\left\|f_{n}(m)-f(m)\right\| \leq\left\|f_{n}-f\right\|_{\infty}+\delta\left\|D f_{n}-D f\right\|_{\infty} \\
=\left\|f_{n}-f\right\|_{C^{1}(\delta)}<\epsilon
\end{aligned}
$$

and hence $\lim _{n \rightarrow \infty} f_{n}(m)=f(m)$, which shows that $f(m)$ is a limit point of $W$ and hence belongs to $W$ as it is a closed set. Since $m \in M$ is arbitrary this shows that $f \in$ $C^{1}(M, W)$ and hence $C^{1}(M, W)$ is closed in $C^{1}\left(M, \mathbb{R}^{N}\right)$.

The following notation will be used in the sequel. Let $D_{N} \subset \mathbb{R}^{N}$ and $D_{d} \subset \mathbb{R}^{d}$ be open subsets and $F \in$ $C^{2}\left(D_{N} \times D_{d}, D_{N}\right)$. The symbols $D_{x} F(\mathbf{x}, \mathbf{z}), D_{z} F(\mathbf{x}, \mathbf{z})$, $D_{x x} F(\mathbf{x}, \mathbf{z})$, and $D_{x z} F(\mathbf{x}, \mathbf{z})$ denote the partial derivatives of $F$ with respect to the variables indicated in the subindices at the point $(\mathbf{x}, \mathbf{z}) \in D_{N} \times D_{d}$.

Lemma E.2 Let $\phi \in \operatorname{Diff}^{1}(M)$ be a dynamical system on the compact differentiable manifold $M$ and consider the observation $\omega \in C^{1}\left(M, \mathbb{R}^{d}\right)$ and state $F \in$ $C^{1}\left(D_{N} \times D_{d}, D_{N}\right)$ maps, with $D_{N} \subset \mathbb{R}^{N}$ and $D_{d} \subset \mathbb{R}^{d}$ open subsets. Let $W \subset D_{N}$ be a subset and suppose that $\omega(M) \subset D_{d}$ and that $F(W \times \omega(M)) \subset W$. Define,

$$
\begin{aligned}
& L_{F_{x}}:=\sup _{(\mathbf{x}, \mathbf{z}) \in W \times \omega(M)}\left\{\left\|D_{x} F(\mathbf{x}, \mathbf{z})\right\|\right\}, \\
& L_{F_{z}}:=\sup _{(\mathbf{x}, \mathbf{z}) \in W \times \omega(M)}\left\{\left\|D_{z} F(\mathbf{x}, \mathbf{z})\right\|\right\} .
\end{aligned}
$$

Suppose that the constants $L_{F_{x}}$ and $L_{F_{z}}$ defined in (E2) are finite and that $L_{F_{x}}\left\|T \phi^{-1}\right\|_{\infty}^{x}<1$. Choose a constant 
$R>0$ such that

$$
R>\frac{L_{F_{z}}\|D \omega\|_{\infty}}{1-L_{F_{x}}\left\|T \phi^{-1}\right\|_{\infty}} .
$$

Then, the map $\Psi$ introduced in (D2) maps the space $C^{1}(M, W)$ into itself and, additionally, it restricts to $\Omega(R, W)$, that is, $\Psi(\Omega(R, W)) \subset \Omega(R, W)$.

Proof. We first note that, for any $f \in C^{1}\left(M, \mathbb{R}^{N}\right)$, its image $\Psi(f)$ belongs necessarily to $C^{1}\left(M, \mathbb{R}^{N}\right)$ as it is a composition of $C^{1}$ maps. Moreover, the hypothesis $F(W \times \omega(M)) \subset W)$ guarantees that $\Psi$ preserves $C^{1}(M, W)$.

We now prove the inclusion $\Psi(\Omega(R, W)) \subset \Omega(R, W)$. Let $f \in \Omega(R, W)$. By the chain rule and the definitions (E2), for any $m \in M$ and $\mathbf{v} \in T_{m} M$ :

$$
\begin{aligned}
& \|D \Psi(f)(m) \cdot \mathbf{v}\| \\
& \begin{array}{l}
\| D_{x} F\left(f\left(\phi^{-1}(m)\right), \omega(m)\right) \cdot D f\left(\phi^{-1}(m)\right) \cdot T_{m} \phi^{-1} \cdot \mathbf{v} \\
\quad+D_{z} F\left(f\left(\phi^{-1}(m)\right), \omega(m)\right) \cdot D \omega(m) \cdot \mathbf{v} \| \\
\leq\left(L_{F_{x}}\|D f\|_{\infty}\left\|T \phi^{-1}\right\|_{\infty}+L_{F_{z}}\|D \omega\|_{\infty}\right)\|\mathbf{v}\| \\
\quad \leq\left(L_{F_{x}} R\left\|T \phi^{-1}\right\|_{\infty}+L_{F_{z}}\|D \omega\|_{\infty}\right)\|\mathbf{v}\|,
\end{array}
\end{aligned}
$$

which, using the hypothesis (E3), implies that

$$
\|D \Psi(f)\|_{\infty} \leq L_{F_{x}} R\left\|T \phi^{-1}\right\|_{\infty}+L_{F_{z}}\|D \omega\|_{\infty}<R,
$$

and guarantees that $\Psi(f) \in \Omega(R, W)$, as required.

$b$. The main theorem. The following result extends Proposition D.1 to the differentiable case. The statement requires an extension of the constants introduced in (E2) to higher order derivatives and with respect to a set $V \subset$ $D_{N}$, that is:

$$
\begin{aligned}
& L_{F_{x}}:=\sup _{(\mathbf{x}, \mathbf{z}) \in V \times \omega(M)}\left\{\left\|D_{x} F(\mathbf{x}, \mathbf{z})\right\|\right\}, \\
& L_{F_{z}}:=\sup _{(\mathbf{x}, \mathbf{z}) \in V \times \omega(M)}\left\{\left\|D_{z} F(\mathbf{x}, \mathbf{z})\right\|\right\}, \\
& L_{F_{x x}}:=\sup _{(\mathbf{x}, \mathbf{z}) \in V \times \omega(M)}\left\{\left\|D_{x x} F(\mathbf{x}, \mathbf{z})\right\|\right\}, \\
& L_{F_{x z}}:=\sup _{(\mathbf{x}, \mathbf{z}) \in V \times \omega(M)}\left\{\left\|D_{x z} F(\mathbf{x}, \mathbf{z})\right\|\right\} .
\end{aligned}
$$

Theorem E.3 Let $\phi \in \operatorname{Diff}^{1}(M)$ be a dynamical system on the compact manifold $M$ and consider the observation $\omega \in C^{1}\left(M, \mathbb{R}^{d}\right)$ and state $F \in C^{2}\left(D_{N} \times D_{d}, D_{N}\right)$ maps, with $D_{N} \subset \mathbb{R}^{N}$ and $D_{d} \subset \mathbb{R}^{d}$ open subsets such that $\omega(M) \subset D_{d}$. Let $V \subset D_{N}$ be a closed convex subset and suppose that $F(V \times \omega(M)) \subset V$. Suppose that the bounds for the partial derivatives of $F$ introduced in (E4) are all finite and that, additionally,

$$
L_{F_{x}}<\min \left\{1,1 /\left\|T \phi^{-1}\right\|_{\infty}\right\} .
$$

Then there exists a compact and convex subset $W \subset V$ such that $F(W \times \omega(M)) \subset W$ and:

(i) The system determined by $F: W \times \omega(M) \longrightarrow W$ and driven by the $\omega$-observations of $\phi$ has the $(\phi, \omega)-E S P$ and hence the generalized synchronization $f_{(\phi, \omega, F)}: M \longrightarrow W$ is well-defined. (ii) The map $f_{(\phi, \omega, F)}$ belongs to $C^{1}(M, W)$ and it is the only one that satisfies the identity (12).

Proof of the theorem. (i) First of all, since by hypothesis $0<L_{F_{x}}<1$, then $F: V \times \omega(M) \longrightarrow V$ is a contraction in the state variable. Indeed, by the Mean Value Theorem and the convexity hypothesis on $V$, for any $\mathbf{x}_{1}, \mathbf{x}_{2} \in V, \mathbf{z} \in \omega(M)$ :

$$
\begin{array}{r}
\left\|F\left(\mathbf{x}_{1}, \mathbf{z}\right)-F\left(\mathbf{x}_{2}, \mathbf{z}\right)\right\| \leq \sup _{\mathbf{x} \in V}\left\{\left\|D_{x} F(\mathbf{x}, \mathbf{z})\right\|\right\}\left\|\mathbf{x}_{1}-\mathbf{x}_{2}\right\| \\
\leq L_{F_{x}}\left\|\mathbf{x}_{1}-\mathbf{x}_{2}\right\| . \quad(\mathrm{E} 6)
\end{array}
$$

The claim then follows from part (i) in Proposition D.1.

(ii) The strategy of the proof consists in showing that, in the hypotheses in the statement, and using the restricted state map $F: W \times \omega(M) \longrightarrow W$ obtained in part (i), the map $\Psi: \Omega(R, W) \longrightarrow \Omega(R, W)$ introduced in (D2) and with $R>0$ satisfying the condition (E3) is a contraction for some norm $\|\cdot\|_{C^{1}\left(\delta_{0}\right)}$ of the type defined in (E1). The value $\delta_{0}>0$ will be specified later on.

This construction is feasible because the hypothesis (E5) implies that $L_{F_{x}}\left\|T \phi^{-1}\right\|_{\infty}<1$. Moreover, by Lemma E.2 and the finiteness of the constants introduced in (E4), a constant $R>0$ can be chosen so that the map $\Psi: \Omega(R, W) \longrightarrow \Omega(R, W)$ is welldefined. Moreover, since by Lemma E.1 the set $\Omega(R, W)$ is closed in $\left(C^{1}\left(M, \mathbb{R}^{N}\right),\|\cdot\|_{C^{1}(\delta)}\right)$ for any $\delta>0$, it is hence a complete metric space to which the Banach Contraction-Mapping Principle [51, Theorem 3.2] can be applied. Consequently, if we are able to prove that $\Psi: \Omega(R, W) \longrightarrow \Omega(R, W)$ is a contraction with respect to the metric inherited from $\left(C^{1}\left(M, \mathbb{R}^{N}\right),\|\cdot\|_{C^{1}\left(\delta_{0}\right)}\right)$, for some $\delta_{0}>0$, we can then conclude the existence of a unique element $f_{0} \in \Omega(R, W)$ such that $\Psi\left(f_{0}\right)=f_{0}$. Now, as we already saw in the proof of part (i), the condition (E5) implies that $L_{F_{x}}<1$ and hence Proposition D.1 guarantees that $f_{(\phi, \omega, F)}: M \longrightarrow W$ is the unique continuous map that satisfies the identity $\Psi\left(f_{(\phi, \omega, F)}\right)=f_{(\phi, \omega, F)}$. Since the element $f_{0} \in \Omega(R, W)$ will be obviously continuous, we shall immediately be able to conclude that $f_{(\phi, \omega, F)}=f_{0}$ and hence that $f_{(\phi, \omega, F)} \in \Omega(R, W) \subset C^{1}\left(M, \mathbb{R}^{N}\right)$, necessarily.

We hence conclude the proof by showing the existence of a constant $\delta_{0}>0$ for which $\Psi: \Omega(R, W) \longrightarrow \Omega(R, W)$ is a contraction in the norm $\|\cdot\|_{C^{1}\left(\delta_{0}\right)}$. Let $f_{1}, f_{2} \in$ $\Omega(R, W)$, and $\delta>0$ arbitrary. By definition,

$$
\begin{gathered}
\left\|\Psi\left(f_{1}\right)-\Psi\left(f_{2}\right)\right\|_{C^{1}(\delta)}=\left\|\Psi\left(f_{1}\right)-\Psi\left(f_{2}\right)\right\|_{\infty} \\
+\delta\left\|D\left(\Psi\left(f_{1}\right)\right)-D\left(\Psi\left(f_{2}\right)\right)\right\|_{\infty} .
\end{gathered}
$$

Since in (E6) we showed that $F: V \times \omega(M) \longrightarrow V$ is a contraction in the state variable with constant $0<$ $L_{F_{x}}<1$ then so is $F: W \times \omega(M) \longrightarrow W$. We can hence conclude using (D3) that

$$
\left\|\Psi\left(f_{1}\right)-\Psi\left(f_{2}\right)\right\|_{\infty}<L_{F_{x}}\left\|f_{1}-f_{2}\right\|_{\infty} .
$$


Let now $m \in M$ and $\mathbf{v} \in T_{m} M$ arbitrary. By the chain rule, the Mean Value Theorem, the convexity of $W$, and the definitions in (E4):

$$
\begin{gathered}
\left\|D \Psi\left(f_{1}\right)(m) \cdot \mathbf{v}-D \Psi\left(f_{2}\right)(m) \cdot \mathbf{v}\right\| \\
=\| D F\left(f_{1}\left(\phi^{-1}(m)\right), \omega(m)\right)\left(D f_{1}\left(\phi^{-1}(m)\right) \cdot T_{m} \phi^{-1} \cdot \mathbf{v}, D \omega(m) \cdot \mathbf{v}\right) \\
-D F\left(f_{2}\left(\phi^{-1}(m)\right), \omega(m)\right)\left(D f_{2}\left(\phi^{-1}(m)\right) \cdot T_{m} \phi^{-1} \cdot \mathbf{v}, D \omega(m) \cdot \mathbf{v}\right) \| \\
\leq \| D F\left(f_{1}\left(\phi^{-1}(m)\right), \omega(m)\right)\left(D f_{1}\left(\phi^{-1}(m)\right) \cdot T_{m} \phi^{-1} \cdot \mathbf{v}, D \omega(m) \cdot \mathbf{v}\right) \\
-D F\left(f_{1}\left(\phi^{-1}(m)\right), \omega(m)\right)\left(D f_{2}\left(\phi^{-1}(m)\right) \cdot T_{m} \phi^{-1} \cdot \mathbf{v}, D \omega(m) \cdot \mathbf{v}\right) \| \\
+\| D F\left(f_{1}\left(\phi^{-1}(m)\right), \omega(m)\right)\left(D f_{2}\left(\phi^{-1}(m)\right) \cdot T_{m} \phi^{-1} \cdot \mathbf{v}, D \omega(m) \cdot \mathbf{v}\right) \\
-D F\left(f_{2}\left(\phi^{-1}(m)\right), \omega(m)\right)\left(D f_{2}\left(\phi^{-1}(m)\right) \cdot T_{m} \phi^{-1} \cdot \mathbf{v}, D \omega(m) \cdot \mathbf{v}\right) \| \\
\leq L_{F_{x}}\left\|\left(D f_{1}\left(\phi^{-1}(m)\right)-D f_{2}\left(\phi^{-1}(m)\right)\right) \cdot T_{m} \phi^{-1} \cdot \mathbf{v}\right\| \\
\quad+\|\left(D_{x} F\left(f_{1}\left(\phi^{-1}(m)\right), \omega(m)\right)\right. \\
\left.-D_{x} F\left(f_{2}\left(\phi^{-1}(m)\right), \omega(m)\right)\right) \cdot\left(D f_{2}\left(\phi^{-1}(m)\right) \cdot T_{m} \phi^{-1} \cdot \mathbf{v}\right) \| \\
\left.\quad \leq D_{z} F\left(f_{1}\left(\phi^{-1}(m)\right), \omega(m)\right)-D z F\left(f_{2}\left(\phi^{-1}(m)\right), \omega(m)\right)\right) \cdot D \omega(m) \cdot \mathbf{v} \| \\
+L_{F_{x x}}\left\|D f_{1}-D f_{2}\right\|_{\infty}\left\|T \phi^{-1}\right\|_{\infty}\left\|D f_{2}\right\|_{\infty}\left\|T \phi^{-1}\right\|_{\infty}\|\mathbf{v}\| \\
\quad+L_{F_{x z}}\left\|f_{1}-f_{2}\right\|_{\infty}\|D \omega\|_{\infty}\|\mathbf{v}\| .
\end{gathered}
$$

If we now use that, by hypothesis, $f_{2} \in \Omega(R, W)$, the last inequality implies that:

$$
\begin{aligned}
\| D \Psi\left(f_{1}\right)(m) & \cdot \mathbf{v}-D \Psi\left(f_{2}\right)(m) \cdot \mathbf{v} \| \\
\leq & \left(L_{F_{x}}\left\|D f_{1}-D f_{2}\right\|_{\infty}\left\|T \phi^{-1}\right\|_{\infty}\right. \\
& +L_{F_{x x}}\left\|f_{1}-f_{2}\right\|_{\infty} R\left\|T \phi^{-1}\right\|_{\infty} \\
& \left.\quad+L_{F_{x z}}\left\|f_{1}-f_{2}\right\|_{\infty}\|D \omega\|_{\infty}\right)\|\mathbf{v}\|,
\end{aligned}
$$

which ensures that

$$
\begin{aligned}
& \left\|D \Psi\left(f_{1}\right)(m) \cdot \mathbf{v}-D \Psi\left(f_{2}\right)(m) \cdot \mathbf{v}\right\| \\
& \leq\left(L_{F_{x x}}\left\|T \phi^{-1}\right\|_{\infty} R+L_{F_{x z}}\|D \omega\|_{\infty}\right)\left\|f_{1}-f_{2}\right\|_{\infty} \\
& \quad+L_{F_{x}}\left\|T \phi^{-1}\right\|_{\infty}\left\|D f_{1}-D f_{2}\right\|_{\infty} .
\end{aligned}
$$

Together with (E1) and (E8), this inequality implies that:

$$
\begin{aligned}
& \quad\left\|\Psi\left(f_{1}\right)-\Psi\left(f_{2}\right)\right\|_{C^{1}(\delta)} \\
& \leq\left(L_{F_{x}}+\delta\left(L_{F_{x x}}\left\|T \phi^{-1}\right\|_{\infty} R+L_{F_{x z}}\|D \omega\|_{\infty}\right)\right)\left\|f_{1}-f_{2}\right\|_{\infty} \\
& \quad+\delta L_{F_{x}}\left\|T \phi^{-1}\right\|_{\infty}\left\|D f_{1}-D f_{2}\right\|_{\infty} . \quad(\mathrm{E} 9)
\end{aligned}
$$

Choose now $\delta_{0}>0$ small enough so that

$$
L_{F_{x}}+\delta_{0}\left(L_{F_{x x}}\left\|T \phi^{-1}\right\|_{\infty} R+L_{F_{x z}}\|D \omega\|_{\infty}\right)<1
$$

which is always possible due to the hypothesis $L_{F_{x}}<1$. Additionally, the hypothesis $L_{F_{x}}\left\|T \phi^{-1}\right\|_{\infty}<1$ together with (E10) and (E9) imply that

$$
\left\|\Psi\left(f_{1}\right)-\Psi\left(f_{2}\right)\right\|_{C^{1}\left(\delta_{0}\right)} \leq c_{0}\left\|f_{1}-f_{2}\right\|_{C^{1}\left(\delta_{0}\right)},
$$

for any $0<c_{0}<1$ such that

$$
\begin{aligned}
c_{0}<\min \left\{L_{F_{x}}\left\|T \phi^{-1}\right\|_{\infty},\right. \\
\left.\quad L_{F_{x}}+\delta_{0}\left(L_{F_{x x}}\left\|T \phi^{-1}\right\|_{\infty} R+L_{F_{x z}}\|D \omega\|_{\infty}\right)\right\},
\end{aligned}
$$

which guarantees the contractivity of $\Psi: \Omega(R, W) \longrightarrow$ $\Omega(R, W)$, as required.

\section{ACKNOWLEDGMENTS}

We thank Edward Ott for pointing out the relation of our research in reservoir computing with the differentiability problem. His observation is at the origin of this contribution. We also thank Herbert Jaeger for his comment about global versus local contractivity as well as the suggestions of the referees that have significantly improved the paper.

$\mathrm{AH}$ is supported by a scholarship from the EPSRC Centre for Doctoral Training in Statistical Applied Mathematics at Bath (SAMBa), project EP/L015684/1. JPO acknowledges partial financial support coming from the Research Commission of the Universität Sankt Gallen, the Swiss National Science Foundation (grant number 200021_175801/1), and the French ANR "BIPHOPROC" project (ANR-14-OHRI-0002-02). The authors thank the hospitality and the generosity of the FIM at ETH Zurich and the Division of Mathematical Sciences of the Nanyang Technological University, Singapore, where a significant portion of the results in this paper were obtained.
[1] L. M. Pecora, T. L. Carroll, G. A. Johnson, D. J. Mar, and J. F. Heagy, Fundamentals of synchronization in chaotic systems, concepts, and applications, Chaos 7, 520 (1997).

[2] E. Ott, Chaos in Dynamical Systems, 2nd ed. (Cambridge University Press, 2002).

[3] S. Boccaletti, J. Kurths, G. Osipov, D. L. Valladares, and C. S. Zhou, The synchronization of chaotic systems, Physics Reports 366, 1 (2002).
[4] D. Eroglu, J. S. W. Lamb, and T. Pereira, Synchronisation of chaos and its applications, Contemporary Physics 58, 207 (2017).

[5] G. Alvarez, S. Li, F. Montoya, G. Pastor, and M. Romera, Breaking projective chaos synchronization secure communication using filtering and generalized synchronization, Chaos, Solitons \& Fractals 24, 775 (2005).

[6] O. I. Moskalenko, A. A. Koronovskii, and A. E. Hramov, Generalized synchronization of chaos for secure commu- 
nication: Remarkable stability to noise, Physics Letters A 374, 2925 (2010).

[7] X. Jiang-Feng, M. Le-Quan, and C. Guan-Rong, A chaotic communication scheme based on generalized synchronization and hash functions, Chinese Physics Letters 21, 1445 (2004).

[8] C. J. Stam, A. M. v. C. van Walsum, Y. A. L. Pijnenburg, H. W. Berendse, J. C. de Munck, P. Scheltens, and B. W. van Dijk, Generalized synchronization of MEG recordings in Alzheimer's disease: evidence for involvement of the gamma band, Journal of Clinical Neurophysiology 19, 562 (2002).

[9] F. Bartolomei, I. Bosma, M. Klein, J. C. Baayen, J. C. Reijneveld, T. J. Postma, J. J. Heimans, B. W. van Dijk, J. C. de Munck, A. de Jongh, K. S. Cover, and C. J. Stam, Disturbed functional connectivity in brain tumour patients: evaluation by graph analysis of synchronization matrices, Clinical Neurophysiology 117, 2039 (2006).

[10] N. F. Rulkov, M. M. Sushchik, L. S. Tsimring, and H. D. I. Abarbanel, Generalized synchronization of chaos in directionally coupled chaotic systems, Physical Review E 51, 980 (1995).

[11] L. Kocarev and U. Parlitz, General approach for chaotic synchronization with applications to communication, Physical Review Letters 74, 5028 (1995).

[12] K. Pyragas, Weak and strong synchronization of chaos, Physical Review E - Statistical Physics, Plasmas, Fluids, and Related Interdisciplinary Topics 54, 4508 (1996).

[13] B. R. Hunt, E. Ott, and J. A. Yorke, Differentiable generalized synchronization of chaos, Physical Review E 55, 4029 (1997).

[14] M. Lukoševičius and H. Jaeger, Reservoir computing approaches to recurrent neural network training, Computer Science Review 3, 127 (2009).

[15] G. Tanaka, T. Yamane, J. B. Héroux, R. Nakane, N. Kanazawa, S. Takeda, H. Numata, D. Nakano, and A. Hirose, Recent advances in physical reservoir computing: A review, Neural Networks 115, 100 (2019).

[16] H. Jaeger and H. Haas, Harnessing Nonlinearity: Predicting Chaotic Systems and Saving Energy in Wireless Communication, Science 304, 78 (2004).

[17] J. Pathak, Z. Lu, B. R. Hunt, M. Girvan, and E. Ott, Using machine learning to replicate chaotic attractors and calculate Lyapunov exponents from data, Chaos 27, 10.1063/1.5010300 (2017), arXiv:1710.07313.

[18] J. Pathak, B. Hunt, M. Girvan, Z. Lu, and E. Ott, ModelFree Prediction of Large Spatiotemporally Chaotic Systems from Data: A Reservoir Computing Approach, Physical Review Letters 120, 24102 (2018).

[19] Z. Lu, B. R. Hunt, and E. Ott, Attractor reconstruction by machine learning, Chaos 28, 10.1063/1.5039508 (2018), arXiv:1805.03362.

[20] T. L. Carroll, Using reservoir computers to distinguish chaotic signals, Physical Review E 98, 52209 (2018).

[21] D. Ibáñez-Soria, J. García-Ojalvo, A. Soria-Frisch, and G. Ruffini, Detection of generalized synchronization using echo state networks, Chaos: An Interdisciplinary Journal of Nonlinear Science 28, 33118 (2018).

[22] T. Weng, H. Yang, C. Gu, J. Zhang, and M. Small, Synchronization of chaotic systems and their machinelearning models, Physical Review E 99, 1 (2019).

[23] T. Lymburn, D. M. Walker, M. Small, and T. Jüngling, The reservoir's perspective on generalized synchronization, Chaos 29, 10.1063/1.5120733 (2019).
[24] F. Takens, Detecting strange attractors in turbulence (Springer Berlin Heidelberg, 1981) pp. 366-381.

[25] J. P. Huke, Embedding nonlinear dynamical systems: a guide to Takens' theorem, Tech. Rep. (Manchester Institute for Mathematical Sciences. The University of Manchester, 2006).

[26] A. G. Hart, J. L. Hook, and J. H. P. Dawes, Embedding and approximation theorems for echo state networks, Neural Networks 128, 234 (2020), arXiv:1908.05202.

[27] M. B. Matthews, On the Uniform Approximation of Nonlinear Discrete-Time Fading-Memory Systems Using Neural Network Models, Ph.D. thesis, ETH Zürich (1992).

[28] M. B. Matthews, Approximating nonlinear fadingmemory operators using neural network models, Circuits, Systems, and Signal Processing 12, 279 (1993).

[29] L. Grigoryeva and J.-P. Ortega, Echo state networks are universal, Neural Networks 108, 495 (2018), arXiv:/arxiv.org/abs/1806.00797 [http:].

[30] L. Gonon and J.-P. Ortega, Reservoir computing universality with stochastic inputs, IEEE Transactions on Neural Networks and Learning Systems 31, 100 (2020), arXiv:1807.02621.

[31] T. Poggio, H. Mhaskar, L. Rosasco, B. Miranda, and Q. Liao, Why and when can deep-but not shallownetworks avoid the curse of dimensionality: A review (2017), arXiv:1611.00740.

[32] N. H. Mhaskar, Neural networks for optimal approximation of smooth and analytic functions, Neural computation 8, 164 (1996).

[33] M. Inubushi and S. Goto, Transfer learning for nonlinear dynamics and its application to fluid turbulence, Physical Review E 102, 43301 (2020).

[34] K. Weiss, T. M. Khoshgoftaar, and D. Wang, A survey of transfer learning, Journal of Big data 3, 1 (2016).

[35] M. P. do Carmo, Riemannian Geometry (Birkhäuser Boston, 1992).

[36] L. Grigoryeva and J.-P. Ortega, Differentiable reservoir computing, Journal of Machine Learning Research 20, 1 (2019).

[37] H. Jaeger, German National Research Center for Information Technology, Tech. Rep. (German National Research Center for Information Technology, 2010).

[38] G. Manjunath and H. Jaeger, Echo state property linked to an input: exploring a fundamental characteristic of recurrent neural networks, Neural Computation 25, 671 (2013), arXiv:1309.2848v1.

[39] G. Manjunath, Stability and memory-loss go hand-inhand: three results in dynamics \& computation, To appear in Proceedings of the Royal Society London Ser. A Math. Phys. Eng. Sci. , 1 (2020), arXiv:2001.00766.

[40] L. Grigoryeva and J.-P. Ortega, Universal discrete-time reservoir computers with stochastic inputs and linear readouts using non-homogeneous state-affine systems, Journal of Machine Learning Research 19, 1 (2018), arXiv:1712.00754.

[41] Z. Lu and D. S. Bassett, Invertible generalized synchronization: A putative mechanism for implicit learning in neural systems, Chaos 30, 10.1063/5.0004344 (2020).

[42] P. Verzelli, C. Alippi, and L. Livi, Learn to Synchronize, Synchronize to Learn, (2020), arXiv:2010.02860.

[43] A. G. Hart, J. L. Hook, and J. H. P. Dawes, Echo State Networks trained by Tikhonov least squares are L2 $(\mu)$ approximators of ergodic dynamical systems, Physica D: 
Nonlinear Phenomena , 132882 (2021).

[44] L. Kocarev and U. Parlitz, Generalized synchronization, predictability, and equivalence of unidirectionally coupled dynamical systems, Physical Review Letters $\mathbf{7 6}$, 1816 (1996).

[45] A. Ceni, P. Ashwin, L. Livi, and C. Postlethwaite, The echo index and multistability in input-driven recurrent neural networks, Physica D: Nonlinear Phenomena 412, 132609 (2020), arXiv:2001.07694.

[46] A. Ceni, P. Ashwin, and L. Livi, Interpreting recurrent neural networks behaviour via excitable network attractors, Cognitive Computation 12, 330 (2020), arXiv:1807.10478.

[47] L. Grigoryeva, A. G. Hart, and J.-P. Ortega, Embedding chaos on manifolds with state-space systems, In preparation (2020).

[48] E. N. Lorenz, Deterministic nonperiodic flow (1963).

[49] L. Gonon, L. Grigoryeva, and J.-P. Ortega, Risk bounds for reservoir computing, Journal of Machine Learning Research 21, 1 (2020).

[50] J. Munkres, Topology, 2nd ed. (Pearson, 2014) p. 503.

[51] J. H. Shapiro, A Fixed-Point Farrago (Springer International Publishing Switzerland, 2016) p. 221.

[52] R. Abraham and J. Robbin, Transversal Mappings and Flows (W. A. Benjamin, Inc, 1967).

[53] M. W. Hirsch, Differential Topology (Springer Verlag, 1976). 